\title{
Metabolic and Functional Response of Neonatal Pig Hearts to the Development of Ischemic Contracture: Is Recovery Possible?
}

\author{
SHONA M. TORRANCE, MICHAEL P. BELANGER, W. JACK WALLEN, AND CARIN WITTNICH \\ Institute of Medical Science [S.M.T, C.W.] and Departments of Surgery [M.P.B., C.W.] and Physiology \\ [W.J.W., C.W.], University of Toronto, Toronto, Ontario M5S 1A8, Canada; and Division of \\ Cardiovascular Surgery, The Hospital for Sick Children, Toronto, Ontario M5G 1X8, Canada [C.W.]
}

\begin{abstract}
ABST
The potential for functional and metabolic recovery in neo-
natal hearts after the development of ischemic contracture re-
mains controversial and undefined. This study documents post-
ischemic recovery of metabolism and function in the in vivo
neonatal heart after the development of onset and peak ischemic
contracture. In piglets on cardiopulmonary bypass, hearts were
reperfused after the development of either onset (TICo) or peak
(TICp) ischemic contracture. Systolic (developed and systolic
function, contractility) and diastolic (diastolic function, relax-
ation) performance was assessed throughout reperfusion. Biop-
sies were obtained at end-ischemia or end-reperfusion to assess
metabolism. By end-ischemia, the metabolic profiles of both
TICo and TICp hearts confirmed energy-store depletion and
purine degradation that was quantitatively greater in TICp hearts.
Hearts reperfused at TICo had consistent moderate impairment
of developed function, contractility, diastolic function, and re-
laxation, whereas hearts reperfused at TICp had much more
profound functional impairment. Diastolic function showed the
worst functional recovery. In contrast, systolic function was not
significantly altered in either study group and, thus, did not
reflect the actual extent of injury. In addition, TICo hearts either
did not further deplete or partially regenerated energy stores
during reperfusion, whereas TICp hearts had further energy-store
\end{abstract}
ABSTRACT

depletion and lactate accumulation. In summary, neonatal hearts reperfused after TICo maintained or partially restored energy stores and had significant but incomplete functional recovery. In contrast, further metabolic deterioration and profound functional impairment occurred with reperfusion after TICp, potentially indicating ongoing mitochondrial injury and compromised oxidative phosphorylation. (Pediatr Res 48: 191-199, 2000)

AXC, aortic cross-clamp

\section{Abbreviations}

$\mathbf{C P}$, creatine phosphate

CPB, cardiopulmonary bypass

$+\mathbf{d P} / \mathbf{d t}_{\text {max }}$, contractility

$\mathbf{- d P / d t}$ max , relaxation

IMP, inosine monophosphate

$\mathbf{L V}$, left ventricle

$\mathrm{PaO}_{2}$, partial pressure of oxygen

SL, sarcolemma

SR, sarcoplasmic reticulum

TAN, total adenine nucleotides

TIC, time to ischemic contracture

TICo, time to ischemic contracture onset

TICp, time to peak ischemic contracture
Whether the neonatal heart is highly tolerant (1) or susceptible (2) to ischemic injury remains controversial. The TIC model has been used to assess myocardial susceptibility to ischemic injury since such landmark papers as those published by Hearse et al. (3) and Wechsler et al. (4). Ischemic contracture, a marker of ischemic injury $(3,4)$, was first recognized clinically when some heart surgery patients developed unrecoverable postischemic myocardial dysfunction $(5,6)$. In adult

Received December 14, 1999; accepted March 12, 2000.

Correspondence: C. Wittnich, D.V.M., Associate Professor Surgery/Physiology, University of Toronto, Clinical Sciences Division, Medical Sciences Building, Rm 7256, Toronto, Ontario M5S 1A8, Canada.

Supported by Grant No. T2454 from the Heart and Stroke Foundation of Ontario. S.M.T. was supported by a Research Fellowship from the Ontario Ministry of Health. hearts, ischemic contracture has been shown to occur secondary to mitochondrial injury (7), altered cytosolic $\mathrm{Ca}^{2+}$ levels $(8)$, and a critical loss of ATP at the myofibrils $(3,9)$ that results in a progressive inability to dissociate actin-myosin cross-bridges (6). Since its recognition and characterization, ischemic contracture has been used as a marker of ischemic tolerance $(3,4)$. Despite the original interest in the TIC model and its metabolic characterization, the functional consequences of and metabolic recovery from ischemic contracture have not been extensively investigated. Doubt has emerged regarding the relevance of TIC in immature hearts (10). As well, the potential for both metabolic and functional recovery with reperfusion after the development of ischemic contracture in neonates has not been fully elucidated. To address these issues, 
the present study compares and contrasts postischemic recovery of myocardial metabolism and function with reperfusion after the development of onset and peak ischemic contracture in the in vivo neonatal heart.

\section{METHODS}

Preparation. In preparation for placement on CPB (11), neonatal male Yorkshire pigs ( $3 \mathrm{~d}$ old, $n=32$ ) were anesthetized with an intraperitoneal injection of sodium pentobarbital $(65 \mathrm{mg} / \mathrm{kg})$. Animals were then intubated and ventilated with medical air. One catheter was inserted into the right carotid artery and advanced to the aortic arch to monitor arterial blood pressure. A second catheter was inserted into the right jugular vein and advanced to the superior vena cava to monitor central venous pressure. These catheters were connected to pressure transducers (COBE, Lakewood, CO, U.S.A.) and a physiologic recorder (BIOPAC Systems, Inc., Goleta, CA, U.S.A.). Arterial blood samples were obtained and appropriate adjustments were made to ensure normal physiologic values for $\mathrm{Po}_{2}$ and $\mathrm{PCO}_{2}$ as well as bicarbonate and pH (ABL30 Acid-Base Analyzer, Radiometer, Copenhagen, Denmark). After sternotomy, systemic heparinization (heparin sulfate, $400 \mathrm{IU} / \mathrm{kg}$ ), and aortic and right atrial cannulation, piglets were placed on normoxic $\left(\mathrm{PaO}_{2}=15.8 \pm 0.8 \mathrm{kPa}\right)$, normothermic $\left(38.3 \pm 0.1^{\circ} \mathrm{C}\right) \mathrm{CPB}$ using a Minimax membrane oxygenator (Medtronic, Anaheim, CA, U.S.A.) and a Sarns 9000 perfusion system (3M SARNS, Ann Arbor, MI, U.S.A.) that was primed with fresh blood. Supplemental sodium pentobarbital was administered via the perfusion circuit to ensure a surgical plane of anesthesia.

Experimental protocol. After the initiation of $\mathrm{CPB}$, a compliant fluid-filled latex balloon was inserted into the LV and connected to a pressure transducer. Heart rate as well as systolic pressure, diastolic pressure, and developed (systolicdiastolic) pressure were obtained directly from the original pressure trace (Fig. 1A). This pressure trace was differentiated with respect to time; contractility and relaxation were defined as the peak positive deflection $\left(+\mathrm{dP} / \mathrm{dt}_{\max }\right)$ and peak negative deflection $\left(-\mathrm{dP} / \mathrm{dt}_{\max }\right)$, respectively (Fig. $\left.1 B\right)$. Myocardial performance was assessed by incrementally injecting fluid into the compliant LV balloon to yield a volume-dependent relationship and to generate performance index-versus-volume curves. For all indices except relaxation (developed function, contractility, systolic function, diastolic function), performance was defined as the slope of the linear portion of the index-versusvolume curve (Fig. 1C). Because relaxation was consistent across this physiologic volume range, relaxation was defined as the mean $-\mathrm{dP} / \mathrm{dt}_{\max }$ value across this volume range. A sample performance index-versus-volume curve is shown from one individual TICo animal (Fig. 2). This varied-volume myocardial performance assessment technique is well accepted in larger animal models and assesses the ability of the heart to respond to a range of volumes, thereby applying the FrankStarling law of the heart to provide more physiologically relevant information.

Preischemic baseline myocardial performance was obtained for all hearts. After confirming stability, global myocardial ischemia was initiated with placement of an AXC. Immedi-

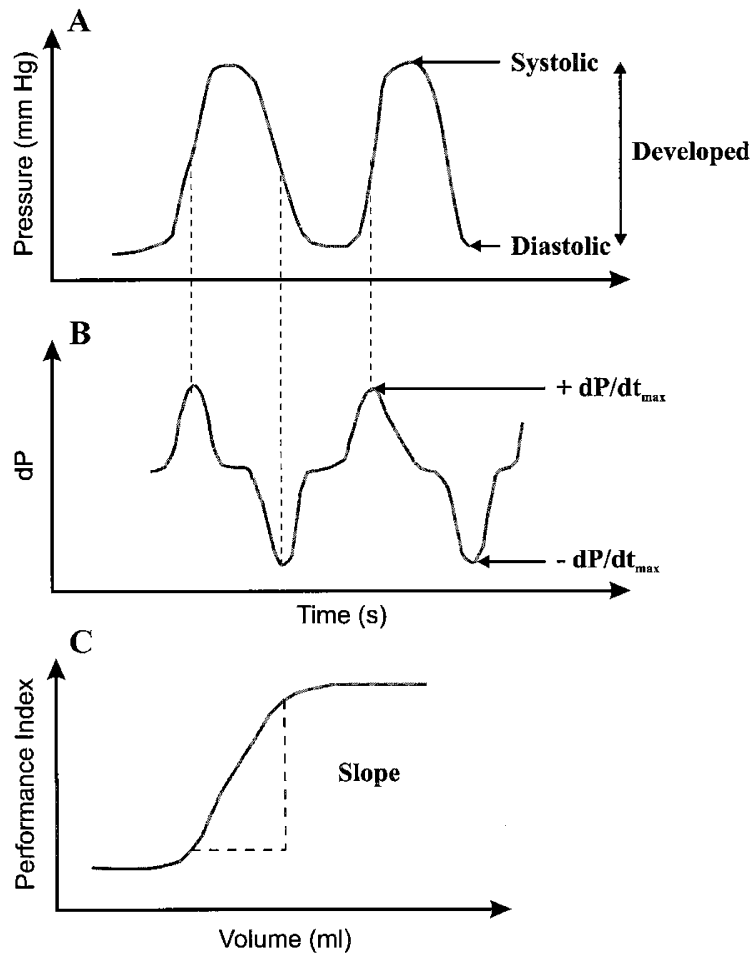

Figure 1. For each balloon volume, the original intraventricular pressure trace $(A)$ yielded pressure values; the first derivative of this trace with respect to time $(B)$ yielded peak maximum $\left(+\mathrm{dP} / \mathrm{dt}_{\text {max }}\right)$ and peak minimum $\left(-\mathrm{dP} / \mathrm{dt}_{\text {max }}\right)$ contractility and relaxation, respectively. For each function assessment, fluid was injected into the balloon to progressively increase the balloon volume, thus generating performance index- $v s$-volume curves $(C)$. Myocardial function was defined as the slope of the linear portion of the index- $v s$-volume curve.

ately after AXC, the LV balloon was filled to generate a pressure of $10 \mathrm{~mm} \mathrm{Hg}$. As the duration of ischemia increased, the pressure in the LV balloon gradually increased, and ischemic contracture onset was defined as a $2-\mathrm{mm} \mathrm{Hg}$ pressure increase, whereas peak ischemic contracture was defined as the pressure plateau. This is a standard technique to assess the development of ischemic contracture (2). The TICo or TICp was measured and recorded for each heart. Due to the potentially deleterious effect of LV biopsies on both coronary perfusion and myocardial function in a neonatal heart model, a biopsy was taken immediately after the development of onset or peak ischemic contracture (end-ischemia) in one subset of hearts $(n=16)$. This biopsy was used to assess the metabolic status at onset or peak ischemic contracture and, thus, determines the metabolic effects of ischemia alone. Postischemic functional recovery could not be assessed in these hearts. In all other hearts $(n=16)$, after reaching either onset or peak ischemic contracture, the fluid was withdrawn from the balloon, the AXC removed, and reperfusion initiated. Reperfusion was confirmed by distension of the main coronary arteries with arterial blood together with the return of normal arterialized color to the myocardium. One TICp heart was excluded from the study due to unsuccessful reperfusion as documented by the lack of coronary distension and an absent LV pressure waveform and confirmed by extremely high diffusible end-product levels (lactate, $157.5 \mu \mathrm{mol} / \mathrm{g}$; hypoxanthine, $4.1 \mu \mathrm{mol} / \mathrm{g}$ ) at 


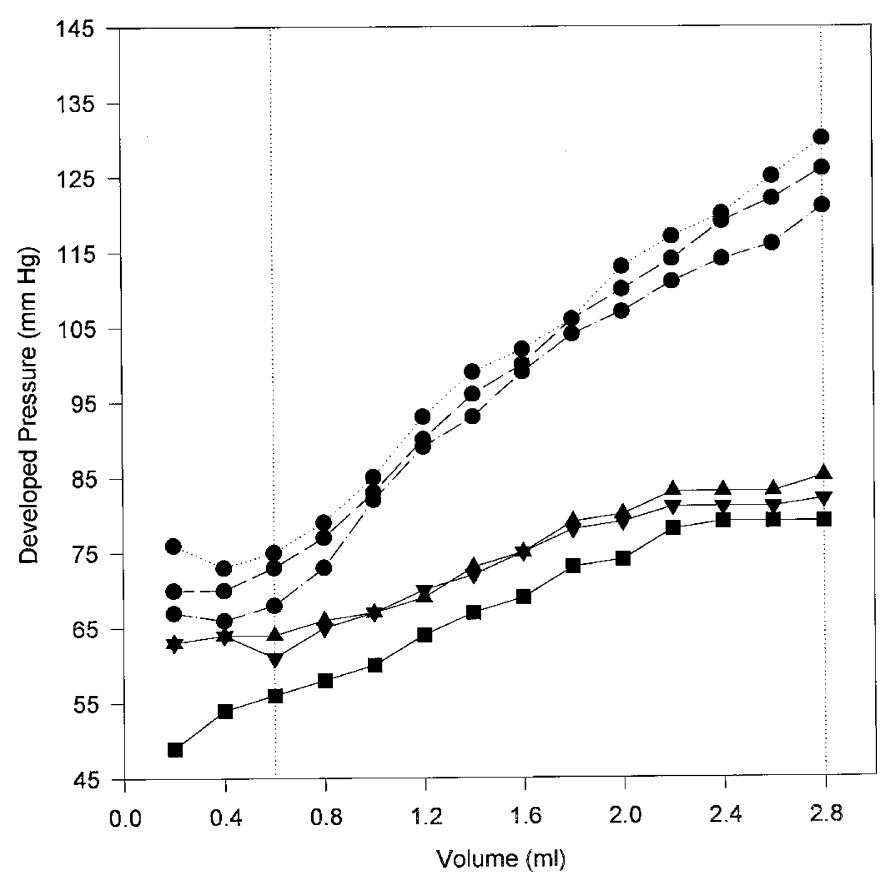

Figure 2. Sample developed pressure- $v s$-volume curves for one TICo heart. In addition to three baseline curves (-), pressure-volume curves are also shown for $15(\boldsymbol{\square}), 30(\boldsymbol{\Delta})$, and $60(\boldsymbol{\nabla})$ min of reperfusion. Although the entire volume range is shown, the slope values are calculated from the linear portion of the range between the dotted lines.

end-reperfusion. In all other animals, postischemic myocardial function was assessed at 15, 30, and 60 min of reperfusion, and a biopsy was obtained at end-reperfusion to determine the combined metabolic effects of ischemia/reperfusion.

After obtaining the LV biopsy at either end-ischemia or end-reperfusion, CPB was terminated and animals were euthanized by pentobarbital overdose. All experimental procedures and protocols used in this investigation were reviewed and approved by the University of Toronto Animal Care and Use Committee and are in accordance with the National Institutes of Health Guide for the Care and Use of Laboratory Animals (National Institutes of Health publication No 85-23, revised 1985) and Canadian Council on Animal Care guidelines.

Myocardial metabolism. Full-thickness freeze-clamp LV free-wall biopsies (12) were immediately immersed in liquid nitrogen, freeze-dried, and stored at $-86^{\circ} \mathrm{C}$ for subsequent analysis. Adenine nucleotides (ATP, ADP, AMP) and nucleosides (hypoxanthine, xanthine, IMP, inosine), pyridine nucleotides (NAD, NADP), and CP were analyzed according to HPLC methods modified by Hull-Ryde et al. (13). Myocardial glycogen and lactate were determined by enzymatic analysis (14, 15). TAN (ATP + ADP + AMP) content was also calculated. Metabolites are expressed as $\mu \mathrm{mol} / \mathrm{g}$ dry weight.

Data analysis. In both study groups, each myocardial performance index was obtained at preischemia and at 15, 30, and $60 \mathrm{~min}$ of reperfusion. Analysis was via univariate repeatedmeasures ANOVA with Duncan's multiple range test post hoc. Group differences in ischemic time and metabolites at endreperfusion were analyzed by $t$ test. For statistical analyses, calculations were performed on the absolute data, and signif- icance was accepted at $p<0.05$. All values are expressed as mean \pm SEM.

\section{RESULTS}

Myocardial metabolism at end-ischemia. In the two study groups, the TICo and TICp were $30.5 \pm 1.9$ and $46.0 \pm 4.0$ min, respectively $(p=0.04)$. At end-ischemia, both study groups had reduced myocardial energy stores with quantitatively greater reductions at peak than onset ischemic contracture. Additionally, ischemia had a quantitatively different impact on the various metabolites. Specifically, both study groups had the least reductions in ATP (Fig. 3A; TICp, $46 \pm 11 \%$, TICo, $13 \pm 6 \%$ ) and greater reductions in CP (Fig. 3B; TICp, $58 \pm 4 \%$, TICo, $55 \pm 9 \%$ ) and glycogen (Fig. 3C; TICp, $78 \pm$ $8 \%$, TICo, $64 \pm 7 \%$ ). Interestingly, TAN levels decreased 17\% in TICp hearts $(22.0 \pm 4.0 \mu \mathrm{mol} / \mathrm{g})$ but remained at baseline levels in TICo hearts $(27.0 \pm 2.5 \mu \mathrm{mol} / \mathrm{g})$.

Myocardial metabolism at end-reperfusion. By endreperfusion, compared with the levels obtained at endischemia, myocardial energy levels were either unaltered or partially restored in the TICo group, whereas those in the TICp group were either unchanged or further reduced. Specifically, at end-reperfusion, the ATP level in the TICo hearts remained $23 \pm 5 \%$ below baseline values but was still 4 .1-fold higher than the $81 \pm 10 \%$ reduction observed in TICp hearts (Fig. $3 D$ ). In contrast, CP levels in the TICo hearts recovered to baseline (Fig. $3 E$ ). This value was 10.3 -fold greater than that seen in the TICp hearts in which CP actually declined further and was $90 \pm 9 \%$ below baseline by end-reperfusion. At end-reperfusion, glycogen in the TICo group partially recovered to $25 \pm 8 \%$ below baseline but remained $68 \pm 21 \%$ below baseline in TICp hearts (Fig. 3F). Despite these differences, large variability in the TICp group prevented statistical significance. Overall, this profile indicates that TICo hearts had much higher myocardial energy stores than TICp hearts by end-reperfusion. In addition, the myocardial pyridine nucleotides at end-reperfusion were significantly higher in TICo hearts with 2.4-fold higher NAD (Fig. 4A; $p=0.01$ ), which approached that seen at baseline, and 2-fold higher NADP (Fig. $4 B ; p=0.02$ ).

In contrast, glycolytic and purine degradation product levels at end-reperfusion were generally higher in the TICp than TICo hearts (Table 1). Specifically, lactate levels at end-reperfusion were 2.4-fold higher in TICp hearts $(p=0.0005)$, and both groups remained elevated from baseline (TICo, $60 \pm 32 \%$ versus TICp, $276 \pm 1 \%$ ). In addition, ADP and AMP remained over $40 \%$ reduced from baseline in both groups with the TICp hearts having 36\% lower ADP levels $(p=0.001)$ and 1.8 -fold higher AMP levels. As a result, compared with baseline, TAN levels at end-reperfusion were reduced $30 \%$ in TICo and $76 \%$ in TICp hearts. Although the diffusible purine degradation products were generally higher in the TICp group, only the 1.7-fold higher hypoxanthine levels achieved statistical significance ( $p=0.02$ ), whereas the 2.4-fold higher IMP yielded a statistical trend $(p=0.08)$.

Postischemic myocardial function. Throughout the study, heart rate was not significantly altered in either study group 

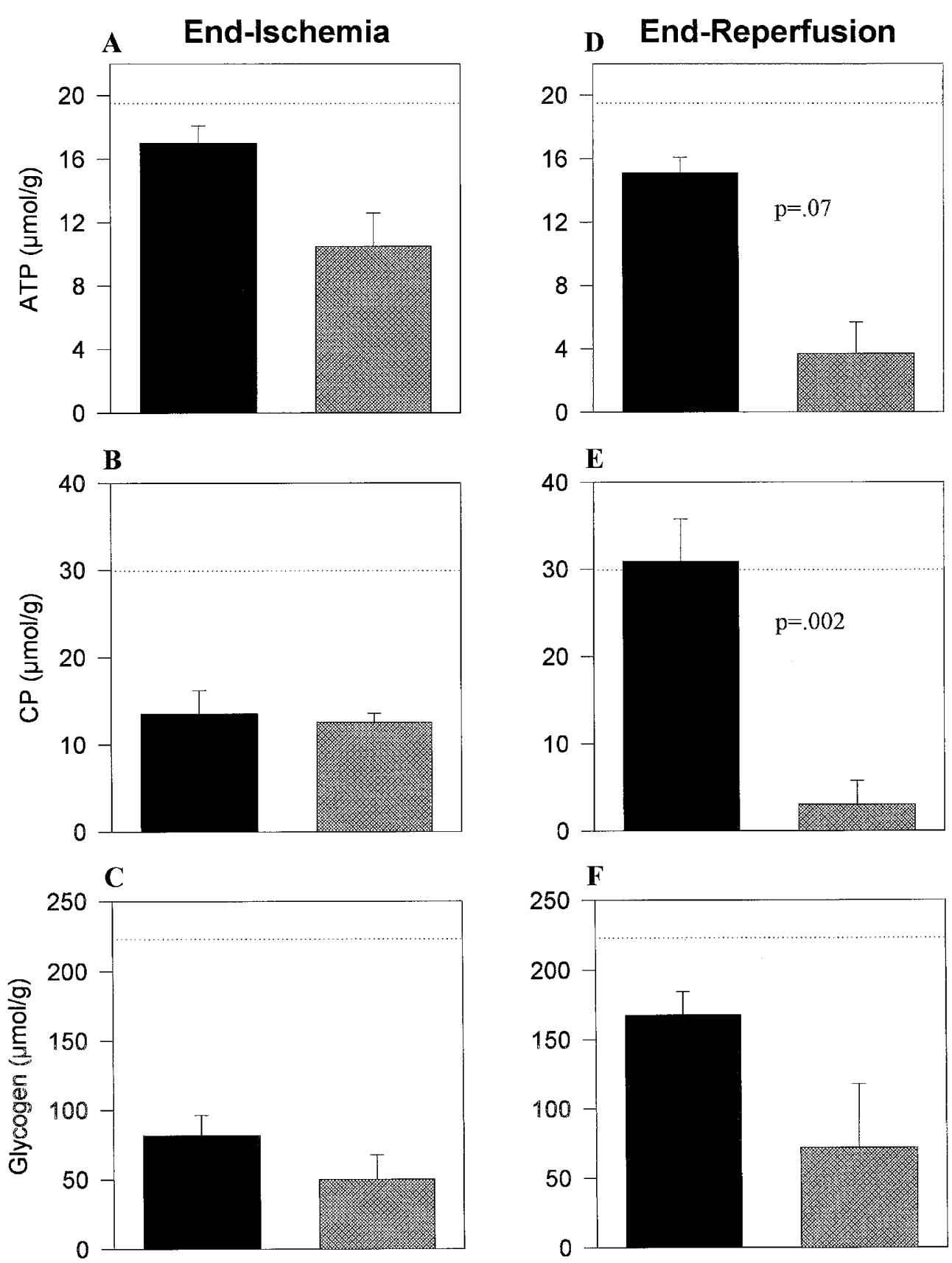

Figure 3. In vivo neonatal myocardial energy stores after the development of onset (solid) or peak (hatch) ischemic contracture at end-ischemia (left) and end-reperfusion (right). Values are mean \pm SEM $(\mu \mathrm{mol} / \mathrm{g}$ dry weight). Values at end-ischemia are shown for ATP $(A)$, CP $(B)$, and glycogen $(C)$. Values at end-reperfusion are shown for ATP $(D), \mathrm{CP}(E)$, and glycogen $(F)$. Normal physiologic values (dotted line) and specific statistical differences at end-reperfusion between onset and peak groups are shown.

$(p=0.70)$, with values of $201 \pm 8$ beats/min at preischemia and $182 \pm 6$ beats/min throughout reperfusion. Absolute values, including baseline, are shown for all systolic performance parameters (Fig. 5). The overall ANOVA statistics for the systolic performance parameters indicate that for all but systolic function, the TICo and TICp groups exhibit significantly different functional recovery with ischemia/reperfusion [developed function $(p<0.0001)$, contractility $(p<0.0001)$, systolic function $(p=0.13)$ ]. Specifically, developed function during reperfusion is shown in Figure $5 \mathrm{~A}$. In the TICo group, developed function was depressed $32 \pm 8 \%(p=0.0004)$ at $15 \mathrm{~min}$ and remained $36 \pm 12 \%(p<0.0001)$ depressed by $60 \mathrm{~min}$ of reperfusion. In contrast, there was significantly worse recovery of developed function after TICp. Specifically, developed function in the TICp group was depressed by $72 \pm 7 \%(p<$ 0.0001 ) at $15 \mathrm{~min}$ and thereafter remained over $80 \%$ depressed $(p<0.0001)$. Contractility during reperfusion in the TICo and TICp groups is shown in Figure $5 B$. In the TICo group, contractility was depressed $36 \pm 11 \%(p<0.0001)$ at $15 \mathrm{~min}$ and did not improve during reperfusion. In contrast, contractility in the TICp group was reduced over 95\% $(p<0.0001)$ throughout the reperfusion period. Systolic function is shown 
A

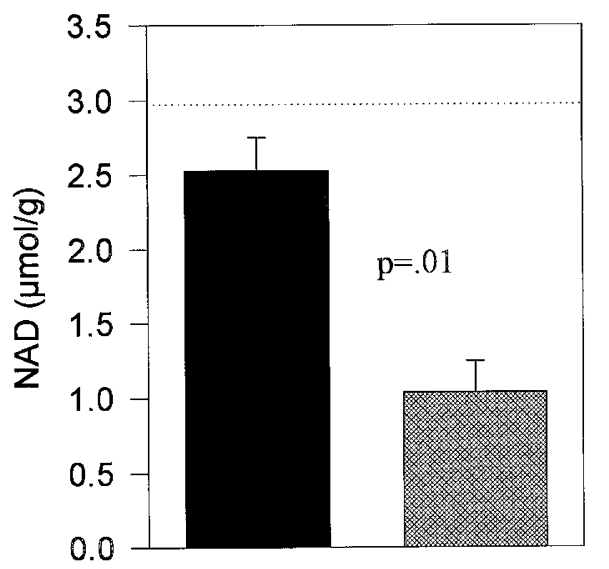

B

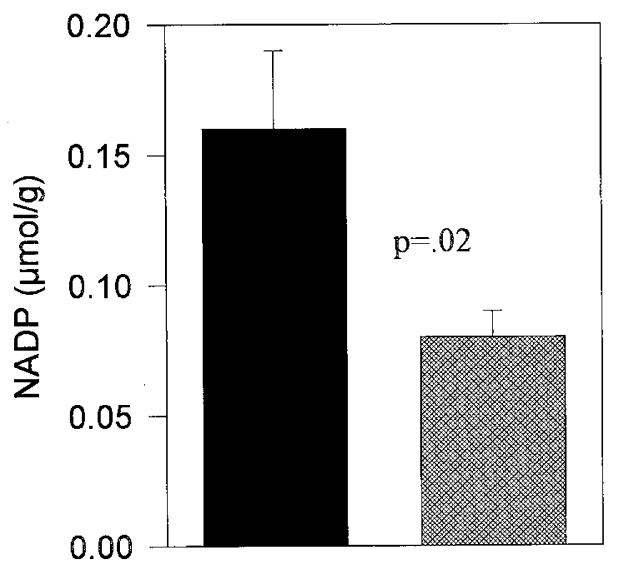

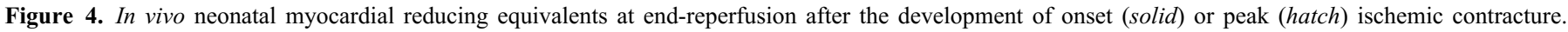

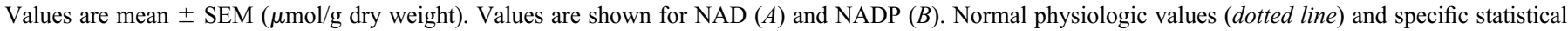
differences between onset and peak groups are shown.

Table 1. Degradation product levels at end-reperfusion

\begin{tabular}{|c|c|c|c|c|}
\hline Metabolite & $\begin{array}{l}\text { Normal } \\
\text { range }\end{array}$ & TICo & TICp & $p$ Value \\
\hline \multicolumn{5}{|c|}{ Purine degradation products } \\
\hline TAN & $26.6 \pm 2.4$ & $18.7 \pm 1.3$ & $6.5 \pm 2.3$ & 0.0002 \\
\hline AMP & $1.15 \pm 0.15$ & $0.39 \pm 0.09$ & $0.72 \pm 0.25$ & NS \\
\hline Hypoxanthine & - & $0.62 \pm 0.09$ & $1.06 \pm 0.08$ & 0.02 \\
\hline Xanthine & - & $0.54 \pm 0.10$ & $0.40 \pm 0.00$ & NS \\
\hline \multicolumn{5}{|c|}{ Glycolytic end-product } \\
\hline Lactate & $14.0 \pm 3.5$ & $22.4 \pm 4.5$ & $52.7 \pm 0.1$ & 0.0005 \\
\hline
\end{tabular}

Values, mean \pm SEM $(\mu \mathrm{mol} / \mathrm{g}$ dry weight). Statistics are between TICo and TICp hearts. NS $=$ not statistically different.

in Figure 5C. The overall ANOVA indicated that systolic function was not significantly altered after ischemia/ reperfusion ( $p=0.13$ ) in either TICo or TICp hearts.

Absolute values for all diastolic performance parameters are shown in Figure 6. The overall ANOVA statistics for the diastolic performance parameters indicate that the TICo and TICp groups had significantly different postischemic functional recovery [diastolic function $(p<0.0001)$, relaxation $(p=$ $0.004)]$. Specifically, diastolic function during reperfusion is shown in Figure $6 \mathrm{~A}$. For this parameter, an increase indicates impaired diastolic function. In the TICo group, diastolic function increased over $80 \%(p<0.05)$ during initial reperfusion and was sustained at this level throughout reperfusion. Diastolic function in the TICp group increased by 3500-4500\% $(p<0.0001)$ throughout the reperfusion period, thus confirming significantly worse diastolic function in TICp hearts than TICo hearts $(p<0.0001)$. Relaxation during reperfusion in the TICo and TICp groups is shown in Figure $6 B$. In the TICo group, relaxation was depressed $33 \pm 9 \%(p<0.0001)$ at 15 min and did not improve throughout reperfusion. In contrast, relaxation in the TICp group was reduced over $80 \%(p<$ 0.0001 ) throughout the reperfusion period.

\section{DISCUSSION}

For the first time, this study quantifies metabolic and functional recovery in neonatal hearts with reperfusion after the development of onset and peak ischemic contracture. Neonatal hearts reperfused after ischemic contracture onset had no further reduction or even partially restored energy stores and slight to moderate reductions in both contractile and diastolic performance. This partial metabolic recovery in individual hearts was consistent with the extent of postischemic functional recovery. In contrast, hearts reperfused after peak ischemic contracture had further reduced energy stores and profound systolic and diastolic dysfunction. Thus, this study documents the extent of postischemic metabolic and functional recovery and definitively disproves the belief that there would be sufficient metabolic and functional recovery to support the circulation in neonatal hearts reperfused after peak ischemic contracture.

Stone heart was first recognized clinically when some heart surgery patients $(5,6)$ had severely compromised postischemic heart function with reperfusion. This occurred due to an increase in diastolic stiffness $(3,5)$ that was initiated during ischemia and was exacerbated with reperfusion. In the current study, the greater impairment in diastolic function also confirms the importance of diastolic stiffness in the neonatal heart. Ischemic contracture occurs secondary to a critical loss of ATP at the myofibrils $(3,9)$, which results in a progressive inability to dissociate actin-myosin cross-bridges (6) and ultimately increases diastolic stiffness $(3,5)$. Since its recognition and characterization, ischemic contracture has been used as a use- 

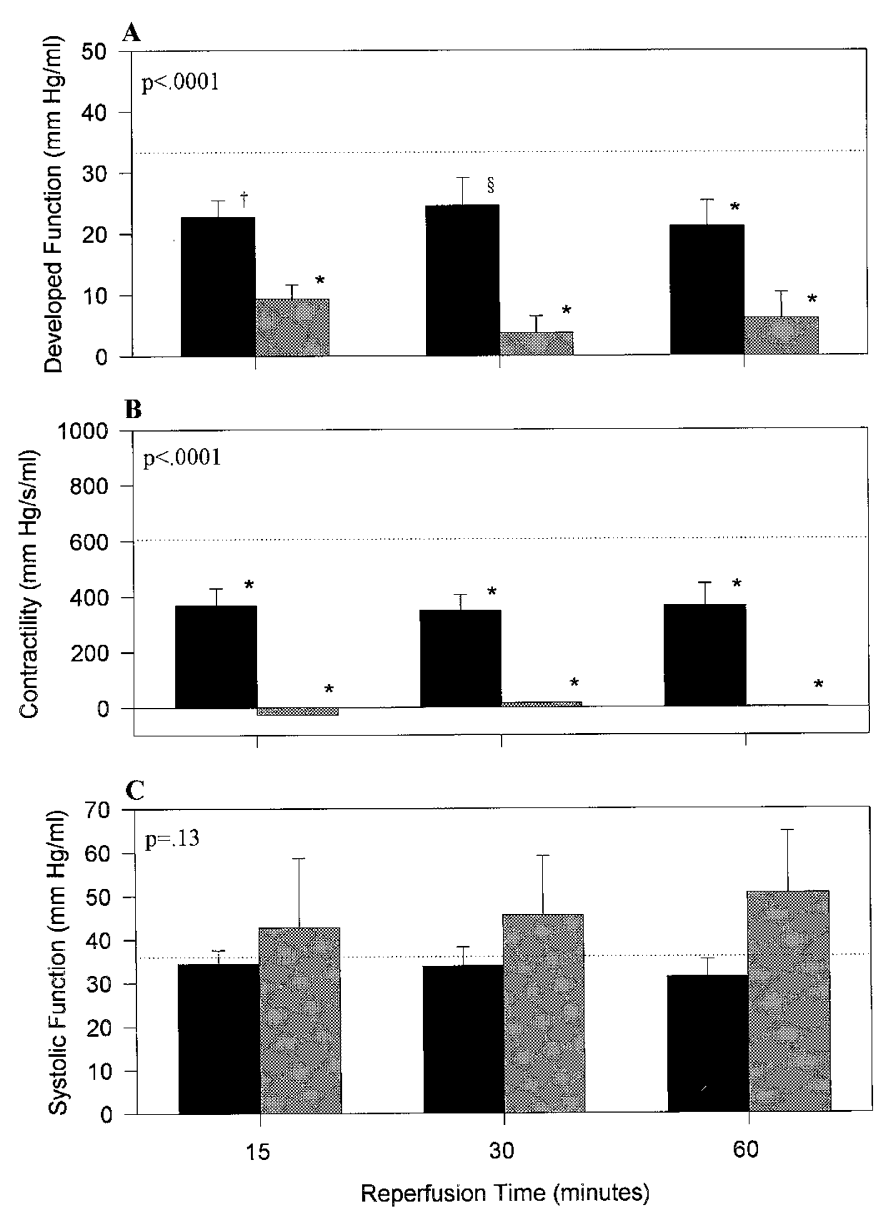

Figure 5. Postischemic systolic performance parameters with reperfusion after the development of onset (solid) or peak (hatch) ischemic contracture. Profiles are shown for developed function $(A)$, contractility $(B)$, and systolic function $(C)$ at 15,30 , and $60 \mathrm{~min}$ of reperfusion. The overall ANOVA statistic and baseline preischemic mean (dotted line) are also shown. Values are mean \pm SEM. Post hoc analysis yielded statistically significant differences from baseline; $* p<0.0001 ; \dagger p<0.0005$; $\$ p<0.005$.

ful marker of ischemic tolerance $(3,4)$, and the TIC model has been used as an index of susceptibility to global ischemic injury $(3,5)$. By use of this model, ischemic damage can be estimated by determining both TICo and TICp. Ischemic contracture onset marks the beginning of rigor complex formation, is characterized by combined reversible and irreversible injury, and is supported by the moderate functional recovery documented in this study. In contrast, peak contracture represents maximal rigor complex formation with extensive irreversible injury and was confirmed by the profoundly impaired postischemic functional recovery observed in all TICp hearts. Both initial (3) and subsequent research $(7,9,16,17)$ on adult TIC focused extensively on the metabolic correlates of onset and peak contracture. Whereas early studies correlated ischemic contracture with ATP depletion in both adults $(7,9,18)$ and newborns (18), more recent studies in adult hearts have identified the cessation of anaerobic glycolysis as the metabolic event that defines the onset of ischemic contracture $(17,19)$. Despite these metabolic studies, postischemic functional recovery after ischemic contracture in the neonatal heart has not been extensively examined. In particular, some researchers
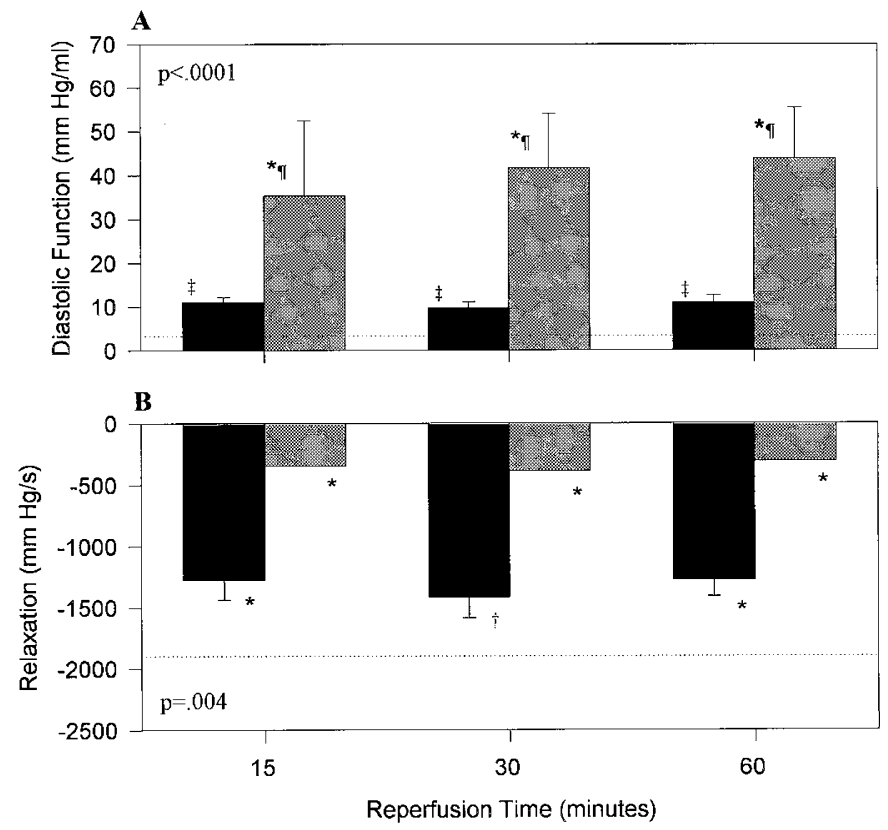

Figure 6. Postischemic recovery of diastolic performance parameters with reperfusion after the development of onset (solid) or peak (hatch) ischemic contracture. Profiles are shown for diastolic function $(A)$ and relaxation $(B)$ at 15, 30, and $60 \mathrm{~min}$ of reperfusion. The overall ANOVA statistic and baseline preischemic mean (dotted line) are also shown. Values are mean \pm SEM. Post hoc analysis yielded statistically significant differences from baseline; ${ }^{*} p<$

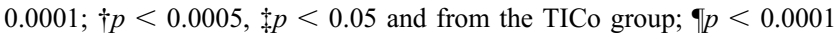

contend that immature hearts will exhibit substantial functional recovery even with reperfusion after TICp. In contrast, the current study shows that neonatal hearts reperfused at TICp had negligible postischemic recovery of most performance indices in addition to severe metabolic compromise.

Traditionally, studies of postischemic functional recovery have used fixed durations of ischemia and, thus, could not provide a direct link between the metabolic events identified in TIC studies and postischemic functional recovery. For example, one study examined ischemic contracture in neonatal hearts but documented TIC in one set of hearts and functional recovery in another set of hearts exposed to fixed durations of ischemia (10). As a result, this study did not examine postischemic functional recovery after the development of ischemic contracture. In fact, because TIC is normally distributed for both adult (16) and newborn (10) hearts, extrapolation of fixed duration data to ischemic contracture should be viewed with caution. The current study confirmed this concern because TICp in some hearts occurred after a shorter duration of ischemia than TICo in other hearts. Thus, the potential for functional recovery after ischemic contracture can only be evaluated in individual hearts reperfused after the development of ischemic contracture. The current study measured TICo or TICp and then evaluated functional recovery in the same heart, thus permitting a direct evaluation of functional recovery after the development of onset or peak ischemic contracture.

\section{Myocardial Metabolism}

Metabolism during ischemia. At end-ischemia, the metabolic profiles for both the TICo and TICp hearts are 
consistent with impaired oxidative phosphorylation. Specifically, at end-ischemia, TICo hearts had modestly depleted energy stores, whereas TICp hearts had much more extensive energy and purine nucleotide depletion. Additionally, the inhibition of glycolysis and the concomitant cessation of glycolytic ATP production have been identified as the metabolic events that define ischemic contracture onset $(17,19)$. Because myocardial glycogen is the only glycolytic substrate available during global ischemia, only a significant reduction in glycogen between onset and peak ischemic contracture would indicate ongoing glycolysis. In this experiment, the similar glycogen levels in TICo and TICp hearts at end-ischemia, despite more profound ATP depletion in TICp hearts, confirms that the cessation of glycolysis also marks ischemic contracture onset in neonatal hearts.

Metabolism during reperfusion. The extent of metabolite depletion during ischemia and the ability to regenerate metabolites during reperfusion both contribute to the myocardial metabolite levels at end-reperfusion. Onset and peak hearts had very different metabolic profiles by endreperfusion. Specifically, at end-reperfusion, the TICo hearts fully restored $\mathrm{CP}$ and partially restored glycogen but had no further significant reduction in ATP and moderately reduced TAN. This could imply that despite the inability to restore ATP to baseline levels, hearts reperfused at TICo regained sufficient mitochondrial function to satisfy their immediate cellular energy requirements. In contrast, even by end-reperfusion, the TICp hearts did not improve their end-ischemic glycogen levels and had additional moderate $\mathrm{CP}$ and ATP reductions with profoundly reduced TAN, consistent with profoundly impaired oxidative phosphorylation and extensive mitochondrial injury. In addition, the elevated diffusible purine nucleoside and lactate levels in TICp hearts suggest that tissue end-product removal was impaired.

The oxidative intermediate profile (NAD, NADP) provides information about cellular reducing equivalents and can also be used to assess metabolic recovery from ischemic contracture. Cytosolic NAD indicates the relative rates of glycolysis and oxidative phosphorylation (20) and is, thus, a marker of cellular energy levels. In TICo hearts, NAD either returned to baseline levels or remained slightly depressed, indicating a modest effect on mitochondrial function. In contrast, the significantly reduced NAD in TICp compared with TICo hearts confirms that mitochondrial function and, thus, oxidative phosphorylation in the TICp hearts were profoundly impaired. Although the supply of reducing equivalents is independent from energy production, NADPH provides a means to shunt reducing equivalents to defend against oxidative stress, thus further compromising cellular energy levels (21). The significantly lower NADP levels in TICp hearts indicate that not only is the supply of reducing equivalents for energy production impaired but also the ability to defend against oxidative injury (22) is likely impaired, potentially further compromising postischemic performance in these neonatal hearts.

\section{Myocardial Function}

Systolic performance. This study found that both TICo and TICp hearts had impaired developed function and contractility, which were quantitatively worse in hearts reperfused at TICp. Interestingly, systolic function was not significantly impaired in either study group. Developed function and contractility depend on many common factors such as troponin $\mathrm{C} \mathrm{Ca}^{2+}$ affinity $(23,24)$ and myofilament $\mathrm{Ca}^{2+}$ sensitivity $(25,26-28)$, which regulate the cross-bridges (29). The consistent 35\% impairment of both developed function and contractility in the TICo hearts throughout reperfusion is in keeping with the fact that they depend on these common mechanisms. However, contractility also depends extensively on the myosin ATPase activity (30) and $\mathrm{SR} \mathrm{Ca}^{2+}$ handling (28), both of which are highly dependent on ATP supply and are adversely affected by acidosis. Within the TICo group, ATP was only minimally reduced, thus indicating that these mechanisms were likely not significantly impaired. This resulted in similar recovery of developed function and contractility, consistent with the observed metabolic recovery in TICo hearts.

In contrast, the TICp hearts had profoundly reduced ATP levels and severe lactate accumulation at end-reperfusion, likely indicating compromised mitochondrial function, impaired cellular perfusion, and tissue acidosis that would contribute to the quantitatively worse recovery of developed pressure and contractility. In addition to impairment of other cellular mechanisms, these hearts likely had severely impaired myosin ATPase activity that would have contributed to the quantitatively greater impairment of contractility than developed function (95 versus $80 \%$ ) in these TICp hearts. The minimal recovery of TICp hearts in the current study suggests that neonatal hearts reperfused after the development of peak ischemic contracture exhibit extensive irreversible injury and yield a level of function that would be incapable of supporting the circulation.

In contrast, systolic function did not appear to be significantly impaired during reperfusion after the development of either onset or peak ischemic contracture. In fact, systolic function in the TICp hearts actually increased by endreperfusion. This may mistakenly be interpreted as sustained or even improved systolic function. However, systolic function is the slope of the systolic pressure-versus-volume relationship and, thus, represents the length-dependent aspect of myofilament deactivation at the end of the contractile phase. The TICo hearts maintained systolic function during ischemia/ reperfusion, suggesting that the systolic pressure-versusvolume relationship was unaltered. In contrast, systolic function appeared to increase slightly in TICp hearts. Because stiffness is the change in pressure for a given change in volume, these systolic data more likely suggest that, even during contraction, the TICp hearts are stiffer during reperfusion. This was confirmed by the diastolic function data.

Diastolic performance. This study also found that both TICo and TICp hearts had impaired diastolic function and relaxation that were quantitatively worse in hearts reperfused at TICp. This diastolic injury indicates increased myocardial stiffness, was evident very early during reperfusion, and did not 
worsen or improve. This myocardial stiffness occurs secondary to greater actin-myosin cross-bridge interactions that are determined largely by the cytosolic $\mathrm{Ca}^{2+}$ levels and regulated by $\mathrm{SR}$ and $\mathrm{SL} \mathrm{Ca}^{2+}$-ATPases. Interestingly, SR and SL $\mathrm{Ca}^{2+}$ ATPase activity depends on sufficient ATP supply but can also be impaired by acidosis. In the TICo group, the consistently impaired diastolic function throughout reperfusion indicates that $\mathrm{Ca}^{2+}$ influx likely occurred with the initiation of reperfusion but was not progressive. This suggests that mitochondrial function supplied sufficient ATP to maintain SR and SL $\mathrm{Ca}^{2+}$. ATPase function and, thus, maintained relatively stable cytosolic $\mathrm{Ca}^{2+}$ levels throughout reperfusion. In contrast, all TICp hearts had significantly elevated lactate at end-reperfusion, likely with concomitant acidosis, and profound ATP depletion that would severely impair cytosolic $\mathrm{Ca}^{2+}$ transport, increase cytosolic $\mathrm{Ca}^{2+}$ levels, and ultimately yield the diastolic injury observed in these hearts. In addition, some individual TICp hearts had progressively worsening diastolic function, indicating progressively impaired ATP-dependent cytosolic $\mathrm{Ca}^{2+}$ regulation during reperfusion.

Relaxation, the change in $-\mathrm{dP} / \mathrm{dt}_{\max }$ for a given change in volume, is dependent on the rate of cytosolic $\mathrm{Ca}^{2+}$ decline, which is determined directly by the rate of $\mathrm{Ca}^{2+}$ transport via the SR and SL $\mathrm{Ca}^{2+}$-ATPases. Both TICo and TICp hearts had significantly impaired relaxation, suggesting that oxidative phosphorylation was insufficient to supply adequate ATP for optimal $\mathrm{Ca}^{2+}$-ATPase function. The quantitatively worse relaxation in the TICp hearts, $80 \%$ impairment versus only $33 \%$ in TICo, is consistent with the profoundly impaired oxidative phosphorylation and extensively reduced myocardial energy stores observed in TICp hearts at end-reperfusion.

\section{Metabolism versus Function}

Within the TICo group as well as between the TICo and TICp groups, the metabolic profiles are consistent with the observed differences in functional recovery. For example, one TICo heart exhibited $115 \%$ recovery of developed function, whereas another TICo heart had only $49 \%$ recovery. These two hearts had the same lactate and glycogen levels, but the heart with better recovery had 49\% higher ATP, 194\% higher CP, $96 \%$ higher NADP, and 38\% higher NAD. This confirms that both high-energy phosphate and reducing equivalent levels are associated with function recovery in the neonatal heart. These profiles are also consistent with the clinical (31) and experimental (32) observation that depletion of myocardial energy stores predisposes neonatal hearts to impaired postischemic functional recovery.

\section{Summary}

This study identified that neonatal hearts subjected to ischemic contracture onset had reduced metabolites during ischemia but had no further reduction or partially restored energy stores during reperfusion and slight to moderate reductions in overall myocardial function. In contrast, reperfusion after peak ischemic contracture further reduced ATP and CP and resulted in profoundly impaired myocardial function. Surprisingly, systolic function did not reflect these metabolic and functional responses and, therefore, is not a useful marker of injury in the neonatal heart. On the basis of all other functional indices examined, reperfusion after ischemic contracture onset would yield cardiac outputs sufficient to support the circulation, whereas reperfusion after peak ischemic contracture in the in vivo neonatal heart would be incompatible with life.

Acknowledgments. The authors thank Dr. P. St. Louis, Ph.D. (clinical chemist), Department of Clinical Biochemistry, The Hospital for Sick Children (Toronto) and C.E. Carlyle for their technical assistance, and M. Libin for his contributions. This work was made possible by $3 \mathrm{M}$ SARNS generous loan of the Sarns 9000 perfusion system, and we acknowledge Bach Simpson Ltd. and Ethicon Ltd. for their contributions.

\section{REFERENCES}

1. Bove EL, Stammers AH 1986 Recovery of left ventricular function after hypothermic global ischemia. J Thorac Cardiovasc Surg 91:115-122

2. Wittnich C, Peniston C, Ianuzzo D, Abel JG, Salerno TA 1987 Relative vulnerability of neonatal and adult hearts to ischemic injury. Circulation 76:V156-V160

3. Hearse DJ, Garlick PB, Humphrey SM 1977 Ischemic contracture of the myocardium: mechanisms and prevention. Am J Cardiol 39:986-993

4. Peyton RB, van Trigt P, Pellom GL, Jones RN, Sink JD, Wechsler AS 1982 Improved tolerance to ischemia in hypertrophied myocardium by pre-ischemic enhancement of adenine triphosphate. J Thorac Cardiovasc Surg 84:11-15

5. Cooley DA, Reul GJ, Wukasch DC 1972 Ischemic contracture of the heart: "stone heart." Am J Cardiol 29:575-577

6. Katz AM, Tada M 1972 The "stone heart." A challenge to the biochemist. Am J Cardiol 29:578-580

7. Sink JD, Currie WD, Pellom GL, Hill RC, Chitwood WR, Wechsler AS 1980 Correlation of mitochondrial function and ischemic contracture. J Thorac Cardiovasc Surg 79:570-578

8. Nayler WG 1981 The role of calcium in the ischemic myocardium. Am J Patho 102:262-270

9. Koretsune Y, Marban E 1990 Mechanism of ischemic contracture in ferret hearts relative roles of $\left[\mathrm{Ca}^{2+}\right]_{\mathrm{i}}$ elevation and ATP depletion. Am J Physiol 258:H9-H16

10. Quantz M, Tchervenkov C, Chiu RCJ 1992 Unique responses of immature hearts to ischemia: functional recovery versus initiation of contracture. J Thorac Cardiovasc Surg 103:927-935

11. Ihnken K, Morita K, Buckberg GD, Ihnken O, Winkelmann B, Sherman M 1997 Prevention of reoxygenation injury in hypoxemic immature hearts by priming the extracorporeal circuit with antioxidants. Cardiovasc Surg 5:608-619

12. Belanger MP, Torrance SM, Panos A, Wittnich C 1992 Multiple in vivo full-thickness myocardial biopsies by freeze-clamping. J Invest Surg 5:143-147

13. Hull-Ryde EA, Lewis WR, Veronee CD, Lowe JE 1986 Simple step gradient elution of the major high-energy compounds and their catabolites in cardiac muscle using high-performance-liquid-chromatography. J Chromatog 377:165-174

14. Passonneau JV, Lowry OH 1993 Enzymatic Analysis. A Practical Guide. Humana Press Inc, Totowa, NJ, pp 105-110

15. Lowry OH, Passonneau JV 1972 Flexible System of Enzymatic Analysis. Academic Press, New York, pp 120-218

16. Jones RN, Attarian DE, Currie WD, Olsen CO, Hill RC, Sink JD, Wechsler AS 1981 Metabolic deterioration during global ischemia as a function of time in the intact normal dog heart. J Thorac Cardiovasc Surg 81:264-273

17. Kingsley PB, Sako EY, Yang MQ, Zimmer SD, Ugurbil K, Foker JE, From AHL 1991 Ischemic contracture begins when anaerobic glycolysis stops: a ${ }^{31} \mathrm{P}-\mathrm{NMR}$ study of isolated rat hearts. Am J Physiol 261:H469-H478

18. Jarmakani JM, Nagatomo T, Langer GA 1978 The effect of calcium and high-energy phosphate compounds on myocardial contracture in the newborn and adult rabbit. J Mol Cell Cardiol 10:1017-1029

19. Vanoverschelde JLJ, Janier MF, Bakke JE, Marshall DR, Bergmann SR 1994 Rate of glycolysis during ischemia determines extent of ischemic injury and functional recovery after reperfusion. Am J Physiol 267:H1785-H1794

20. Lee AC, Xu X, Colombini M 1996 The role of pyridine dinucleotides in regulating the permeability of the mitochondrial outer membrane. J Biol Chem 271:2672426731

21. Kehrer JP, Lund LG 1994 Cellular reducing equivalents and oxidative stress. Free Radic Biol Med 17:65-75

22. Bolli R 1991 Oxygen-derived free radicals and myocardial reperfusion injury: an overview. Cardiovasc Drugs Ther 5:249-269

23. Endoh M 1998 Changes in intracellular $\mathrm{Ca}^{2+}$ mobilization and $\mathrm{Ca}^{2+}$ sensitization as mechanisms of action of physiological interventions and inotropic agents in intact myocardial cells. Jap Heart J 39:1-44

24. Fuchs F, Wang YP 1997 Length-dependence of actin-myosin interaction in skinned cardiac muscle fibres in rigor. J Mol Cell Cardiol 29:3267-3274 
25. Babu A, Sonnenblick EH, Gulati J 1988 Molecular basis for the influence of muscle length on myocardial performance. Science 240:74-76

26. Allen DG, Kentish JC 1985 The cellular basis of the length-tension relation in cardiac muscle. J Mol Cell Cardiol 17:821-840

27. Lakatta EG 1987 Starling's law of the heart is explained by an intimate interaction of muscle length and myofilament calcium activation. J Am Coll Cardiol 10:1157-1164

28. Pieske B, Schlotthauer K, Schattman J, Beyersdorf F, Martin J, Just H, Hasenfuss G $1997 \mathrm{Ca}^{2+}$-dependent and $\mathrm{Ca}^{2+}$-independent regulation of contractility in isolated human myocardium. Basic Res Cardiol 92(suppl 1):75-86

29. Winegard S 1984 Regulation of cardiac contractile proteins. Circ Res 55:566-574
30. Roos KP 1997 Mechanics and force production. In: Langer GA (ed) The Myocardium. Academic Press, San Diego, CA, pp 235-323

31. Hammon JW, Graham TP, Boucek RJ, Parrish MD, Merrill WH, Bender HW 1987 Myocardial adenosine triphosphate content as a measure of metabolic and functional myocardial protection in children undergoing cardiac operation. Ann Thorac Surg 44:467-470

32. Wittnich C, Maitland A, Vincente W, Salerno T 1991 Not all neonatal hearts are equally protected from ischemic damage during hypothermia. Ann Thorac Surg 52:1000-1004 